\title{
Evaluation of Visual Field Progression in Glaucoma
}

\author{
Angelo P. Tanna $\cdot$ Rajen U. Desai
}

Published online: 7 May 2014

(C) Springer Science + Business Media New York 2014

\begin{abstract}
Detection of visual field progression is of critical importance in the management of patients with glaucoma; however, there is no agreed upon set of criteria for the definition of progression. The most commonly utilized method of progression detection in clinical practice may be subjective assessment of serial visual fields. Event-based and trend-based approaches to the analysis of serial visual field data are quite powerful and used in clinical practice and in clinical research. Recent advances in analysis of visual field progression are discussed in this review article.
\end{abstract}

Keywords Glaucoma · Visual field P Perimetry ·

Ophthalmology

\section{Introduction}

Determining the presence and rate of visual field (VF) progression is a critical component in the management of glaucoma, affecting the decision to initiate, intensify or advance therapy. In spite of its importance, there is no widely agreed upon standardized set of criteria for judging progression. A recent systematic review disclosed that in all Cochrane reviews and protocols related to glaucoma interventions, visual field progression or change was reported in 13 different ways [1]. Moreover, it is well known that there is only fair agreement among some of the various methods for the determination of visual field progression $[2,3,4 \bullet \bullet, 5]$.

A. P. Tanna $(\bowtie) \cdot$ R. U. Desai

Department of Ophthalmology, Northwestern University

Feinberg School of Medicine, 645 N. Michigan Ave., Suite 440,

Chicago, IL 60611, USA

e-mail: atanna@northwestern.edu
Even the best of efforts to detect subtle progression can be confounded by factors including suboptimal and variable patient reliability, progressive media opacity and the long-term fluctuation that is an inherent aspect of visual psychophysics. Glaucoma experts themselves often disagree on the determination of progression when presented with the same VF series [6].

Numerous groups of investigators have recently reported findings that significantly impact upon clinical practice and clinical research with respect to the use of perimetry to monitor glaucoma. In this article, we will review some of these major advances in our understanding of fluctuation as a confounding factor in the detection of visual field progression, the importance of monitoring the central visual field, the use of the size V stimulus in advanced glaucoma, novel enhancements of pointwise linear regression analysis, frequency-doubling technology perimetry, the visual field index and the concept of combining structural and functional assessments in the detection of glaucoma progression.

\section{Fluctuation in Threshold Sensitivity}

When one tests the threshold sensitivity at a particular location in the visual field, one must anticipate a certain degree of variation or fluctuation from test to test. This is called long-term fluctuation. This variability is the major challenge to the sensitive and specific detection of visual field progression. It has long been known that the more severely damaged the visual field is at baseline, the greater the degree of anticipated future fluctuation [7]. Many factors contribute to the magnitude of threshold sensitivity fluctuation including the overall degree of visual field damage, the degree of damage at the particular location 
being tested, the eccentricity of a particular testing location (the farther from fixation the greater the fluctuation) and other factors.

As a specific example, the variability in the mean deviation (MD) is inversely correlated with the baseline MD itself. That is, the worse the MD (more negative) is, the greater the degree of variation in the MD with retesting. New data based on computer simulations also show the pattern of overall visual field damage is a factor that determines the magnitude of fluctuation in MD. Eyes with global diffuse damage tend to have more variability than eyes with focal damage despite having similar baseline MD values [8].

Wall et al. [9] investigated the relationship of MD and its variability when testing is performed with Goldmann size III and size V stimuli. They determined that the repeatability of size V MD was slightly better than size III, but variability increased with increasing damage for both stimuli [10]. For patients with poor acuity and/or severe visual field damage, the use of standard automated perimetry (SAP) with the size $\mathrm{V}$ stimulus extends the period of time computerized perimetry can be used to monitor the disease process.

In general terms, there are two broad approaches to the analysis of visual field data that are used to detect progression: (1) event-based methods and (2) trend-based methods. Event-based methods define progression as having occurred once some pre-determined degree of deterioration has taken place. Trend-based methods assess whether there is a statistically significant downward trend in some particular measure of the visual field, such as the mean deviation.

\section{Event-Based Progression Analysis}

Guided Progression Analysis (GPA; Carl Zeiss Meditec, Inc., Dublin, CA) is the most important example of an event-based system in widespread clinical use. GPA uses essentially the same criteria used to define progression in the Early Manifest Glaucoma Trial (EMGT) [11]. By performing repeat visual field testing six times over the course of about 1 month in about 100 stable glaucoma patients, the EMGT investigators developed a database that was used to estimate the amount of expected fluctuation at a given location in the visual field at different levels of baseline damage severity.

A study comparing GPA analysis with the majority expert consensus by a group of glaucoma specialists found fair agreement, with GPA being more conservative in declaring progression compared to the experts [4*0]. In cases in which GPA results were discordant with expert consensus, the experts usually classified the visual field series as showing progression. A follow-up study found that in such discordant cases, subsequent visual fields were more likely to convert to the GPA classification of likely progression compared to control series in which GPA and expert consensus was no progression (Tanna AP and Budenz DL, unpublished data). The major limitations of GPA are its failure to detect progression when it occurs diffusely throughout much of the central $24^{\circ}$ of the visual field and its inability to detect progression in severe disease.

\section{Trend-Based Progression Analysis}

Examples of trend-based analyses include linear regression of the mean deviation (MD) or visual field index (VFI). Regression analysis of MD, for example, relates visual function to time by means of a straight-line equation, but is limited because it does not distinguish between focal and diffuse change from either glaucoma progression or media opacity.

\section{Pointwise Linear Regression}

Pointwise linear regression analysis (PLR) is a type of trend-based analysis that can be performed with software such as PROGRESSOR (Institute of Ophthalmology, University College London, and Moorfields Eye Hospital National Health System Foundation Trust, London, UK). This method utilizes raw threshold sensitivity data to determine the slope of change at each testing location in the visual field as a function of time and also yields the statistical significance of the change at each location. Two problems arise with this technique. First, PLR does not yield a global $p$ value of likelihood of the presence of true progression. Second, it is increasingly clear that clinicians must consider the rate of change in the management of patients with glaucoma. PLR yields numerous rates of change for the visual field, one for each testing location. Knowledge about the global rate of change based on PLR would be useful for clinicians.

O'Leary et al. [12] developed a statistical technique that involves permutation analysis to provide a global rate of change based on PLR that they have named permutation of PLR (PoPLR). Karakawa et al. [13] applied the binomial test to the results of PLR to derive a summary value that yields the rate of deterioration of the visual field as a whole.

The standard criteria for the determination of progression at a given location of the visual field using PLR has traditionally been a decline in threshold sensitivity of $1 \mathrm{~dB} /$ year with a $p<0.01$. A recent study by Kummet et al. [14] suggests that an apparently higher degree of sensitivity 
(hit rate) can be achieved while maintaining a high specificity by further restricting the slope criterion to about $-1.2 \mathrm{~dB} /$ year and relaxing the significance level criterion to $p<0.04$. In this study, these criteria resulted in a specificity of $\geq 0.90$ and identified approximately $35 \%$ of glaucoma patients as progressing, a figure in line with other studies of visual field progression, suggesting an appropriate level of sensitivity for progression detection [14].

Another approach to enhance PLR for glaucoma management involves a method to adjust for spatial correlation based on the optic disc and retinal nerve fiber layer anatomy. This approach seems to minimize the effect of outlier locations that show deterioration or improvement due to artifact [15].

Although not frequently used in routine clinical practice, PLR is quite powerful and worthy of more widespread use. The above-cited advances can potentially be combined to develop commercially available software that can be used to aid in the detection of visual field progression.

\section{Visual Field Index}

The visual field index (VFI) was designed to be a more accurate summary measurement of the visual field status than the mean deviation. The VFI a summary statistic of a single visual field test as a percent of the normal ageadjusted sensitivity, center-weighted to correlate with ganglion cell density [16].

The VFI calculation algorithm uses the pattern deviation plot to identify abnormal locations and then uses the total deviation data to calculate the severity of the abnormality at each identified location. This approach mitigates the impact of media opacity of the VFI result. Once the MD falls below $-20 \mathrm{~dB}$, however, only the total deviation plots are used for both identification of abnormal locations and grading the severity of the damage at each location. Accordingly, when MD falls below $-20 \mathrm{~dB}$, there can be a sudden change in the VFI that can be misinterpreted to mean there has been a substantial change in the rate of visual field loss, whereas this is only an artifact of the VFI methodology [17].

One study that compared pointwise event analysis (GPA) with VFI and MD trend analysis showed only fair agreement between GPA and the two approaches to trend analysis [18]. This is not surprising because GPA tends to detect progression more quickly in the early stages of the disease and GPA tends to fail or take longer to detect progression in eyes with diffuse progression or very severe damage. Analysis of VFI trends is not useful for early glaucoma unless numerous visual field tests are available for analysis, primarily because of the small effect of early loss on global index values such as VFI. The two different types of analyses are better used together in making a determination as to whether progression has occurred and whether the rate of deterioration is clinically meaningful.

\section{Assessment of the Central Visual Field in Glaucoma}

Automated perimetry of the central $10^{\circ}$ has recently been shown to be of particular importance in eyes with initial parafoveal scotomas in the setting of glaucoma [19•]. In their important study, Park et al. retrospectively studied 50 eyes followed with longitudinal 10-2 and 24-2 perimetry and detected progression in 24 eyes with 10-2 visual fields using point-wise linear regression. Among these eyes, progression was missed in 17 using the 24-2 visual fields. Conversely, progression was detected in 11 eyes using the 24-2 fields. Among these eyes, 10-2 fields failed to detect progression in only 4. Because of the likely importance of progression of parafoveal progression on visual function and quality of life, these findings suggest patients with parafoveal scotomas should be followed with both 10-2 and 24-2 SAP. We typically follow such patients with both testing algorithms, alternating between them from visit to visit.

In another study from the same group, Su et al. [20] analyzed the patterns of central visual field progression in eyes with initial parafoveal scotoma. They show the initial defects are really arcuate in nature and deepen and spread toward either nasally or toward the physiologic blind spot. As progression occurs, these scotomas initially spare the area corresponding to the papillomacular bundle.

In addition to monitoring eyes with initial parafoveal scotomas, central visual field testing is also important in eyes with severe damage in which much of the central $24-30^{\circ}$ of the field is so depressed that only the central field can be meaningfully monitored for progression. In both types of situations, the determination of progression based on evaluation of the central $10^{\circ}$ of the visual field is problematic, as commercially available perimetry platforms do not include either event- or trend-based analyses to aid in the detection of progression with central visual field testing algorithms.

De Moraes et al. [21] recently developed and validated a set of criteria that uses PLR analysis of the threshold sensitivity data obtained with 10-2 Humphrey visual field tests to detect progression. These criteria include the slopes and statistical significance of changes in sensitivity over time. Uniquely, an additional criterion for progression also takes into account the locations of clusters of progressing testing locations within seven possible sectors within the central $10^{\circ}$ of the visual field. These sectors are defined based on the fact they include testing locations that are highly correlated with respect to the fact they progress at similar frequencies and rates [21]. 


\section{Frequency Doubling Technology Perimetry}

Although controversial, there is increasing evidence of the utility of frequency doubling technology perimetry (FDT) for the early detection of glaucoma $[22,23]$. There are no established criteria or commercially available tools for the detection of FDT VF progression. A recent report of a large cohort of subjects with suspected glaucoma at baseline selected from the Diagnostic Innovations in Glaucoma and the African Descent and Glaucoma Evaluation studies demonstrated that, on average, FDT pattern standard deviation (PSD) deteriorated earlier than SAP PSD and that rates of FDT PSD change were predictive of SAP VF progression, whereas the rates of SAP PSD change were not significant predictors of FDT progression during follow-up [24]. Although FDT PSD trends proved to be statistically significantly greater in eyes that later developed SAP conversion to glaucoma, there was considerable overlap in FDT PSD slope in progressing and non-progressing eyes.

Conversely, in a cohort of glaucoma subjects and normal controls monitored for a median of about 5 years, progression was identified in fewer glaucoma patients with FDT compared with SAP using permutation analyses of PLR of either total deviation or pattern deviation data [25]. About one-third of control subjects were identified as having deterioration with SAP, using total deviation data, but in only $8 \%$ with FDT. This was likely due to progressive cataract and true deterioration in visual function with advancing age. Though small, this study suggests FDT is not particularly more useful for detection of glaucoma progression among patients with established glaucomatous optic neuropathy at baseline.

There are no commercially available progression detection algorithms for FDT perimetry, and there is no reference database that reveals test-retest variability as a function of testing location and severity of baseline damage, as there are for SAP. This limits the utility of FDT perimetry for the longitudinal management of glaucoma in routine clinical practice.

\section{Combining Structure and Function}

Medeiros et al. used both structural data derived from spectral domain OCT and visual field data to calculate a novel "retinal ganglion cell (RGC) index." Expanding on the work of Harwerth et al. [26, 27••], the authors showed that a simple approach of combining readily available structural and functional test data resulted in an index value that performed better than either structural or functional measures alone for progression detection in a wide spectrum of glaucoma severity $[28,29]$.
Structural measures of progression using peripapillary RNFL thickness data tend to underestimate progression in advanced glaucoma, while functional measures perform better and vice versa. Accordingly, the RGC index weights functional measures of progression more heavily in late stage disease and emphasizes structural assessment in early glaucoma [28, 29].

\section{Summary}

The detection of visual field progression continues to be a challenging area of glaucoma management. Increasingly, it is clear that rates of change are very important; therefore, the use of trend-based analyses will likely be more heavily utilized in conjunction with event-based methods. Of equal importance is the concept that techniques that combine structural and functional measures of progression are more powerful and yield more accurate determinations of progression across a spectrum of disease severity.

Acknowledgments This paper is supported by an unrestricted grant from Research to Prevent Blindness, New York, NY.

Disclosure Angelo P. Tanna and Rajen U. Desai have no conflicts of interest to declare.

Human and Animal Rights and Informed Consent This article does not contain any studies with human or animal subjects performed by any of the authors.

\section{References}

Papers of particular interest, published recently, have been highlighted as:

- Of importance

•- Of major importance

1. Ismail R, Azuara-Blanco A, Ramsay CR. Outcome measures in glaucoma: a systematic review of cochrane reviews and protocols. J Glaucoma. 2013.

2. Katz J, Congdon N, Friedman DS. Methodological variations in estimating apparent progressive visual field loss in clinical trials of glaucoma treatment. Arch Ophthalmol. 1999;117(9):1137-42.

3. Iester M, Capris E, De Feo F, Polvicino M, Brusini P, Capris P, Corallo G, Figus M, Fogagnolo P, Frezzotti P, Manni G, Perdicchi A. Agreement to detect glaucomatous visual field progression by using three different methods: a multicentre study. $\mathrm{Br}$ J Ophthalmol. 2011;95(9):1276-83.

4. •- Tanna AP, Budenz DL, Bandi J, Feuer WJ, Feldman RM, Herndon LW, Rhee DJ, Whiteside-de Vos J, Huang J, Anderson DR. Glaucoma progression analysis software compared with expert consensus opinion in the detection of visual field progression in glaucoma. Ophthalmology. 2012;119(3):468-73. The authors demonstrates that GPA is more conservative in declaring progression compared to majority glaucoma expert consensus. 
5. Rao HL, Kumbar T, Kumar AU, Babu JG, Senthil S, Garudadri CS. Agreement between event-based and trend-based glaucoma progression analyses. Eye (Lond). 2013;27(7):803-8.

6. Tanna AP, Bandi JR, Budenz DL, Feuer WJ, Feldman RM, Herndon LW, Rhee DJ, Whiteside-de Vos J. Interobserver agreement and intraobserver reproducibility of the subjective determination of glaucomatous visual field progression. Ophthalmology. 2011;118(1):60-5.

7. Heijl A, Lindgren A, Lindgren G. Test-retest variability in glaucomatous visual fields. Am J Ophthalmol. 1989;108(2):130-5.

8. Russell RA, Garway-Heath DF, Crabb DP. New insights into measurement variability in glaucomatous visual fields from computer modelling. PLoS One. 2013;8(12):e83595. doi:10. 1371/journal.pone.0083595.

9. Wall M, Doyle CK, Eden T, Zamba KD, Johnson CA. Size threshold perimetry performs as well as conventional automated perimetry with stimulus sizes III, V, and VI for glaucomatous loss. Invest Ophthalmol Vis Sci. 2013;54(6):3975-83.

10. Wall M, Kutzko KE, Chauhan BC. Variability in patients with glaucomatous visual field damage is reduced using size $\mathrm{V}$ stimuli. Invest Ophthalmol Vis Sci. 1997;38(2):426-35.

11. Heijl A, Leske MC, Bengtsson B, Hussein M. Measuring visual field progression in the Early Manifest Glaucoma Trial. Acta Ophthalmol Scand. 2003;81:286-93.

12. O'Leary N, Chauhan BC, Artes PH. Visual field progression in glaucoma: estimating the overall significance of deterioration with permutation analyses of pointwise linear regression (PoPLR). Invest Ophthalmol Vis Sci. 2012;53(11):6776-84.

13. Karakawa A, Murata H, Hirasawa H, Mayama C, Asaoka R. Detection of progression of glaucomatous visual field damage using the point-wise method with the binomial test. PLoS One. 2013;8(10):e78630. doi:10.1371/journal.pone.0078630.

14. Kummet CM, Zamba KD, Doyle CK, Johnson CA, Wall M. Refinement of pointwise linear regression criteria for determining glaucoma progression. Invest Ophthalmol Vis Sci. 2013;54(9): 6234-41.

15. Betz-Stablein BD, Morgan WH, House PH, Hazelton ML. Spatial modeling of visual field data for assessing glaucoma progression. Invest Ophthalmol Vis Sci. 2013;54(2):1544-53.

16. Bengtsson B, Heijl A. A visual field index for calculation of glaucoma rate of progression. Am J Ophthalmol. 2008;145(2):343-53.

17. Rao HL, Senthil S, Choudhari NS, Mandal AK, Garudadri CS. Behavior of visual field index in advanced glaucoma. Invest Ophthalmol Vis Sci. 2013;54(1):307-12.

18. Nassiri N, Moghimi S, Coleman AL, Law SK, Caprioli J, NouriMahdavi K. Global and pointwise rates of decay in glaucoma eyes deteriorating according to pointwise event analysis. Invest Ophthalmol Vis Sci. 2013;54(2):1208-13.
19. • Park SC, Kung Y, Su D, et al. Parafoveal scotoma progression in glaucoma: Humphrey 10-2 versus 24-2 visual field analysis. Ophthalmology. 2013;120:1546-50. The authors demonstrate that parafoveal scotomas may be better followed with both 10-2 and 24-2 fields, instead of only the latter.

20. Su D, Park SC, Simonson JL, et al. Progression pattern of initial parafoveal scotomas in glaucoma. Ophthalmology. 2013;120:520-7.

21. de Moraes CG, Song C, Liebmann JM, Simonson JL, Furlanetto RL, Ritch R. Defining 10-2 visual field progression criteria: exploratory and confirmatory factor analysis using pointwise linear regression. Ophthalmology. 2013. doi:10.1016/j.ophtha. 2013.10.018.

22. Liu S, Yu M, Weinreb RN, Lai G, Lam DS, Leung CK. Frequency-doubling technology perimetry for detection of the development of visual field defects in glaucoma suspect eyes: a prospective study. JAMA Ophthalmol. 2014;132(1):77-83.

23. Nakagawa S, Murata H, Saito H, Nakahara H, Mataki N, Tomidokoro A, Iwase A, Araie M. Frequency doubling technology for earlier detection of functional damage in standard automated perimetry-normal hemifield in glaucoma with low-tonormal pressure. J Glaucoma. 2012;21(1):22-6.

24. Meira-Freitas D, Tatham AJ, Lisboa R, Kuang TM, Zangwill LM, Weinreb RN, Girkin CA, Liebmann JM, Medeiros FA. Predicting progression of glaucoma from rates of frequency doubling technology perimetry change. Ophthalmology. 2014;121(2): 498-507. doi:10.1016/j.ophtha.2013.09.016.

25. Redmond T, O'Leary N, Hutchison DM, Nicolela MT, Artes PH, Chauhan BC. Visual field progression with frequency-doubling matrix perimetry and standard automated perimetry in patients with glaucoma and in healthy controls. JAMA Ophthalmol. 2013;131(12):1565-72.

26. Harwerth RS, Wheat JL, Fredette MJ, Anderson DR. Linking structure and function in glaucoma. Prog Retin Eye Res. 2010;29(4):249-71.

27. •• Marvasti AH, Tatham AJ, Zangwill LM, Girkin CA, Liebmann JM, Weinreb RN, Medeiros FA. The relationship between visual field index and estimated number of retinal ganglion cells in glaucoma. PLoS One. 2013;8(10):e76590. The authors demonstrate the utility of a novel retinal ganglion cell index combining data points from $\mathrm{OCT}$ and perimetry.

28. Medeiros FA, Lisboa R, Weinreb RN, Girkin CA, Liebmann JM, Zangwill LM. A combined index of structure and function for staging glaucomatous damage. Arch Ophthalmol. 2012;130(9):1107-16.

29. Medeiros FA, Lisboa R, Weinreb RN, Girkin CA, Liebmann JM, Zangwill LM. A combined index of structure and function for staging glaucomatous damage. Arch Ophthalmol. 2012;130(5):E1-10. 